\title{
The Recurrence Rate in Meningiomas: Analysis of Tumor Location, Histological Grading, and Extent of Resection
}

\author{
Konstantinos Violaris, Vasileios Katsarides, Pavlos Sakellariou \\ Department of Neurosurgery, G. Papanikolaou Hospital, Thessaloniki, Greece \\ Email:kaviola@hotmail.com
}

Received November 20, 2011; revised December 8, 2011; accepted December 22, 2011

\begin{abstract}
Background: Purpose of this study was to evaluate various parameters of meningioma after surgical treatment and analyze predictive factors for recurrence. Methods: During 1992-2007, 353 patients were operated for intracranial meningioma in our department. They were followed since, and related data were combined with parameters such as tumor histology (WHO system), tumor location and the extent of tumor resection (Simpson's scale). The results were analyzed with respect to tumor reappearance. This study was approved by the ethics committee of G. Papanikolaou Hospital. Results: The mean follow up period was 6.45 years. The overall percentage of recurrence was $21.52 \%$. Grade 1 meningiomas (benign) recurred at a rate of $19.1 \%$, grade 2 tumors (atypical) showed $41.7 \%$ rate of recurrence and grade 3 meningiomas (malignant) recurred at a rate of $75 \%$. Tumor histopathology was not significant to recurrence $(\mathrm{p}>0.001)$. The location of meningiomas was not found to be significant to recurrence $(\mathrm{p}>0.001)$. Complete tumor resection was accomplished in 269 patients (76.2\%). Incomplete resection (Simpson grades 2-5) took place in 84 cases (23.8\%). The recurrence rate after complete resection was $13.8 \%$, while the rate for cases with incomplete resection was $46.7 \%$. The extent of removal was significantly associated with recurrence $(\mathrm{p}<0.001)$. Conclusions: Tumor recurrence was observed at $21.5 \%$ of patients in our series of intracranial meningioma surgery. The rate of recurrence was related primarily to the extent of surgical removal. Neither tumor site, nor tumor histology were predictive factors for recurrence (malignant meningiomas excluded).
\end{abstract}

Keywords: Intracranial Meningioma; Histology; Resection Extent; Recurrence

\section{Introduction}

Meningiomas occur at any point of the central nervous system, arising from the arachnoid cap cells of the dura matter [1,2]. They are considered to be benign, slowgrowing, well circumscribed tumors. Unfortunately, after surgical resection, recurrence of these tumors frequently appears [1-3]. This often leads to reoperation and raise of morbidity and mortality risk for the patients. Recurrence is usually associated with factors such as malignancy, subtotal tumor resection or even tumor site [1,2].

Objective of this study was to evaluate the postoperative behavior of the meningioma and to assess whether the tumor's location, histopathological subtype and extent of surgical resection are predictive factors for recurrence. We present the results from our series on the outcome of meningioma patients, in a 15 year period. Parameters such as morbidity and mortality, multiple recurrence, multiple operations, use of radiotherapy, shuntdepended hydrocephalus after surgery are considered. Perioperative complications are also presented.

\section{Clinical Material and Methods}

An observational retrospective study took place over a period of 15 years, from 1992 to 2007 . The study comprised 353 patients, treated by the staff of Thessaloniki G. Papanikolaou General Hospital neurosurgical department. This study was approved by the ethics committee of G. Papanikolaou Hospital. Hospitalization files, medical charts and neuroradiological images obtained for the patients with meningioma, were analyzed with respect to clinical, operative and laboratory pathological data. Tumor histological classification was conducted by the institute's pathologists. Postoperative follow up examinations, carried out by the department's neurosurgeons were analyzed for this study. Sufficient data were available for 275 patients.

Follow up examinations: The endpoint for recurrence was given by a computerized tomography scan (CT) or magnetic resonance image (MRI), showing a meningioma in a location contiguous with the previous surgery. The patients underwent follow up examination and 
imaging at 3 and 6 months after surgery and then every year. They were followed via clinical examination or by telephone interview. Living patients interviewed by telephone described their symptoms referable to brain tumor. Relatives of deceased reported if death had occurred due to tumor related complications or by unrelated causes. The Karnofsky scale was used to evaluate the patients' outcome after the operation.

Histopathological study: Meningiomas were divided into subtypes based on World Health Organization criteria. In our series, the following histopathological subtypes appeared:

Grade 1: meningothelial, fibrous, psammomatus, transitional, stroviloid, epithilioid, angiomatus microcytic, secretory, chordoid

Grade 2: atypical

Grade 3: malignant

Completeness of resection: For evaluating resection, Simpson's scale of grading the extent of surgical removal was used [1]. This scale divides the extent of tumor resection in 5 grades:

Grade 1: complete removal

Grade 2: complete removal with coagulation of dural attachment

Grade 3: complete removal, without coagulation of dural attachment or resection of involved sinus or hyperostotic bone

Grade 4: subtotal resection

Grade 5: decompression-biopsy

For patients of grades 4 and 5, endpoint for recurrence was enlargement of the remaining tumor, shown on MRI or CT.

Statistical analysis: The SPSS system (version 15.0.1) was used for statistical analysis of the experiment's results data. Analysis for descriptive statistics for each variable was conducted. Quality controls for normality, means and variances were also done.

\section{Results}

\subsection{General Results}

The average patient's age at the time of meningioma treatment was 57 years \pm 6 years. The mean follow up period was 6.45 years. The overall percentage of recurrence was $21.52 \%$ (59 patients). Recurrence rate for three, five and ten year period was $22.9 \%, 23 \%$ and $25.5 \%$ respectively. Twelve patients recurred multiply (3.4\%). Nine of them underwent a second operation. Six patients recurred for a third time and were reoperated. Finally, 4 patients underwent 4 craniotomies.

Post surgery, three-year survival rate was $86.3 \%$. The rate for five-year survival was $74.3 \%$ and the ten-year percentage of survival was $66.7 \%$.

Twelve perioperative and five intraoperative deaths were observed. Most common cause for perioperative mortality was pulmonary embolism (seven patients). Five patients after craniotomy developed shunt-depended hydrocephalus. Eight patients without total resection of the tumor underwent radiotherapy after the operation (three of them with meningiomas of histological grading II and III).

Three patients developed a metastasis at locations such as the thoracic spinal cord, the parotid gland and a different site of the brain. The idea of multiple meningiomatosis was excluded, because there was no sign of the new meningioma when the pre-operative MRI was conducted. All three of them had a meningioma with malignant histological characteristics.

Age was not found to affect the appearance of recurrence. Although sometimes surgeons tend to be more conservative when it comes to elderly patients, according to our results, age did not affect the extent of surgical removal, as defined by Simpson, and consequently was not a predictive factor for recurrence $(p>0.01)$.

The majority of our patients were females (220 patients, $62.3 \%$ ), who recurred at a rate of $19 \%$. Males presented recurrence at a percentage of $25.5 \%$.

The majority $(55.2 \%)$ of recurrences was observed within two years from surgery, and $94.7 \%$ within five years from surgery. The relation of recurrence and time after surgery is shown on Table 1.

The morbidity and mortality outcomes were higher for patients without recurrence, as shown on Table 2.

Twenty three patients with recurrence experienced tumor-related death and the rates of 3,5 and 10-year survival were higher for the patients without recurrence.

\subsection{Tumor Site}

Most commonly, parasagital (32.3\%), convexity (23.8\%), tentorium (12.4\%) and sphenoid wing (9.6\%) meningiomas were accounted. Recurrence was observed in $23 \%$ of parasagital meningiomas, $16.9 \%$ of convexity meningiomas, $17.5 \%$ of tentorial and $19.4 \%$ of sphenoid wing meningiomas. The rate of tumor site appearance and their percentages of recurrence are given in Table 3. Tumor location was not significantly associated with recurrence $(\mathrm{p}>0.01)$.

Table 1. Appearance of recurrence in relation with time after operation.

\begin{tabular}{cc}
\hline Time of recurrence: & Number of patients: \\
\hline $0-12$ months & $21(27.2 \%)$ \\
$1-2$ years & $21(27.2)$ \\
$2-3$ years & $15(19.7 \%)$ \\
$3-5$ years & $15(19.7 \%)$ \\
$5-10$ years & $3(4.0 \%)$ \\
More than 10 years & $1(1.3 \%)$ \\
\hline
\end{tabular}


Table 2. Patient outcome in relation with tumor recurrence.

\begin{tabular}{lll}
\hline Patient's status: & $\begin{array}{l}\text { Patients without } \\
\text { recurrence: }\end{array}$ & $\begin{array}{l}\text { Patients with } \\
\text { recurrence: }\end{array}$ \\
\hline 3-year survival & $190(88.5 \%)$ & $46(78.5 \%)$ \\
5-year survival & $168(78.4 \%)$ & $36(60.8 \%)$ \\
10 year survival & $151(69.7 \%)$ & $34(57.7 \%)$ \\
Karnofsky score $>80$ & $139(64.3 \%)$ & $12(20.3 \%)$ \\
Karnofsky score $<80$ & $20(9.2 \%)$ & $5(8.4 \%)$ \\
Death of another reason & $37(17.1 \%)$ & $6(10.1 \%)$ \\
Perioperative death & $4(1.8 \%)$ & $1(1.7 \%)$ \\
Postoperative death & $10(4.6 \%)$ & $2(3.4 \%)$ \\
Death related to the tumor & 0 & $23(38.9 \%)$ \\
\hline
\end{tabular}

Table 3. Tumor location and recurrence.

\begin{tabular}{lll}
\hline Tumor site & Number of patients & Recurrence rate \\
\hline Parasagital & $104(32.3 \%)$ & $23.1 \%$ \\
Convexity & $77(23.8 \%)$ & $16.9 \%$ \\
Tentorial & $40(12.4 \%)$ & $17.5 \%$ \\
Sphenoid wing & $31(9.6 \%)$ & $19.4 \%$ \\
Cerebellopontine angle & $16(5 \%)$ & $37.5 \%$ \\
Olfactory groove & $13(4.3 \%)$ & $7.1 \%$ \\
Orbital & $10(3.1 \%)$ & $0 \%$ \\
Cerebellum convexity & $8(2.5 \%)$ & $25 \%$ \\
Planum sphenoidale & $7(2.2 \%)$ & $28.6 \%$ \\
Petroclival & $5(1.5 \%)$ & $60 \%$ \\
Optic nerve sheath & $4(1.2 \%)$ & $50 \%$ \\
Tuberculum sellae & $4(1.2 \%)$ & $100 \%$ \\
Cavernous sinus & $2(0.6 \%)$ & $100 \%$ \\
Pineal & $1(0.3 \%)$ & $100 \%$ \\
\hline
\end{tabular}

Total resection is often difficult because of structures meningiomas at certain sites (such as clivus, optic nerve sheath, tuberculum sellae, cavernous sinus) embrace. In our series, non total resection took place at a percentage of $59.4 \%$ in operations concerning the referred sites, while surgeries conducted at other cranial locations accomplished total resection at a rate of $84.5 \%$. Never-theless, these sites account for only $6.9 \%$ of the cases in our series. The recurrence rate for these patients was $56 \%$.

\subsection{Tumor Histology}

The rates of histological type appearance and their percentages of recurrence are presented in Table 4. Most commonly, the meningothenial (32\%), fibrous $(14.5 \%)$, psammomatus (14.5\%) and transitional subtype (13.5\%) were accounted in our series. The rates of recurrence for these benign subtypes were $20.5 \%, 25 \%, 15 \%$ and $27 \%$, respectively. The rate of recurrence for the cohort of grade 1 meningiomas in our series was $19.1 \%$.
Table 4. Histology and recurrence.

\begin{tabular}{lll}
\hline $\begin{array}{l}\text { Tumor histologic } \\
\text { subtype: }\end{array}$ & $\begin{array}{l}\text { Number of } \\
\text { patients: }\end{array}$ & $\begin{array}{l}\text { Percentage of } \\
\text { recurrence: }\end{array}$ \\
\hline Meningothelial & $88(32 \%)$ & $20.5 \%$ \\
Fibrous & $40(14.5 \%)$ & $25 \%$ \\
Psammomatus & $40(14.5 \%)$ & $15 \%$ \\
Transitional & $37(13.5 \%)$ & $27 \%$ \\
Stroviloid & $12(4.4 \%)$ & $16.7 \%$ \\
Epithilioid & $8(2.9 \%)$ & $12.5 \%$ \\
Angiomatus & $7(2.5 \%)$ & $28.6 \%$ \\
Microcytic & $6(2.2 \%)$ & $33.3 \%$ \\
Secretory & $4(1.5 \%)$ & $50 \%$ \\
Chordoid & $4(1.5 \%)$ & $25 \%$ \\
Atypical (gr.II) & $12(4.4 \%)$ & $41.7 \%$ \\
Malignant (gr.III) & $12(4.4 \%)$ & $75 \%$ \\
\hline
\end{tabular}

Tumor histological classification was not significant to recurrence $(\mathrm{p}>0.01)$.

Meningiomas of histological grading 2 and 3 accounted for $9.5 \%$ of all tumors in our series (24 patients). The follow up control for patients with non-benign meningiomas revealed that grade 3 meningiomas recurred at a rate of $75 \%$ and grade 2 meningiomas at a rate of $41.6 \%$.

Survival rates for three, five and ten year observation post surgery were significantly lower for these rare subtypes, compared to benign meningiomas. Analysis follows on Table 5.

The morbidity and mortality outcomes were lower for grades 2 and 3 than for grade 1 meningiomas. Six patients with malignant meningioma and two with atypical meningioma experienced tumor-related death. Three patients with malignant meningioma developed a metastasis.

\subsection{Resection Extend (According to Simpson's Scale)}

Complete resection was accomplished in 269 patients (76.2\%). The recurrence rate after complete resection was $13.8 \%$.

Incomplete resection (Simpson grades 2, 3, 4 and 5) was performed in 84 patients $(23.8 \%)$. Tumors with grade 2 resection (complete resection with coagulation of the affected dura) recurred at a rate of $29.6 \%$. Tumors with grade 3 resection (complete resection of tumor, without coagulation of the dura or removal of affected sinus or bone) recurred at a percentage of $56.3 \%$, while $51.3 \%$ of grade 4 patients developed tumor enlargement. The entire recurrence rate for cases with subtotal resection was $46.7 \%$. The extent of tumor removal was significantly associated with recurrence $(\mathrm{p}<0.001)$. The results are presented in Table 6. 
Table 5. Benign, atypical and malignant meningiomas outcome.

\begin{tabular}{cccc}
\hline $\begin{array}{c}\text { Tumor } \\
\text { Subtype }\end{array}$ & $\begin{array}{c}3 \text {-year } \\
\text { survival rate }\end{array}$ & $\begin{array}{c}5 \text {-year } \\
\text { survival rate }\end{array}$ & $\begin{array}{c}\text { 10-year } \\
\text { survival rate }\end{array}$ \\
\hline Benign & $86.6 \%$ & $74.5 \%$ & $67.2 \%$ \\
Atypical & $66.6 \%$ & $58.3 \%$ & $33.3 \%$ \\
Malignant & $33.3 \%$ & $8.3 \%$ & $0 \%$ \\
\hline
\end{tabular}

Table 6. Recurrence in relation with tumor resection extend (Simpson's scale).

\begin{tabular}{ccc}
\hline Resection extent & Number of patients & Percentage of recurrence \\
\hline Grade 1 & 269 patients $(76.2 \%)$ & $13.8 \%$ \\
Grade 2 & 27 patients (7.6\%) & $29.6 \%$ \\
Grade 3 & 16 patients (4.5\%) & $56.3 \%$ \\
Grade 4 & 39 patients (11\%) & $51.3 \%$ \\
Grade 5 & 2 patients $(0.6 \%)$ & $100 \%$ \\
\hline
\end{tabular}

\section{Discussion}

Although meningiomas are considered to be benign tumors, recurrence is observed frequently, with rates that vary between series $[1,4,5]$. After a surgical resection that appears total under the operating microscope, recurrence rate is still high.

The best accepted factor for prediction of recurrence is the 1957 Simpson grading system for completeness of resection which evaluated invasion of the venous sinuses, tumor nodules in adjacent dura, and infiltration of bone by meningothelial cells as chief causes for recurrence [1]. Simpson classified the extent of resection as: Grade 1, complete removal; Grade 2, complete removal with coagulation of dural attachment; Grade 3; complete removal, without coagulation of dural attachment or resection of involved sinus or hyperostotic bone; Grade 4, subtotal resection; and Grade 5, decompression-biopsy.

The recurrence rates that Simpson refers to were 9\% for grade $1,16 \%$ for grade 2, $29 \%$ for grade 3, 39\% for grade 4 and $100 \%$ for grade 5 , respectively.

Since then, others have used the Simpson grading system to analyze their series and came up with results suggesting that there were more factors provoking recurrence to be considered [4-9]. Remaining neoplastic dural cells around the site of craniotomy were proposed as one of these factors [4]. Another study supports that remaining meningotheliomatous cells on dural strips could be the reason for some cases of recurrence, as these cells were not found in patients without meningiomas [5]. Another important factor for predicting recurrence is peritumoral brain edema [6]. Edema is possibly related with meningioma aggressiveness. Vascular endothelial growth factor has been associated with recurrence appearance, due to increase of neovascularization [7]. Another possible factor for recurrence is the increased number of mitosis on histopathological examination, which is related with tumor malignancy [7]. Indicators of cellular multiplication, such as Budr (bromodeoxyuridine), MIB-1/Ki67 and MMP-9 (Matrix metalloproteinase) have been associated with meningioma malignancy $[8,9]$. Some surgeons support that tumor cells left in the arachnoid membrane are responsible for the tumor's reappearance [10].

The percentages of recurrence are not alike in all studies, as shown on Table 7.

In our series, the overall percentage of recurrence was $21.5 \%$. The rate of recurrence for three years post operation was $22.9 \%$, for five years $23 \%$ and for ten years $25.5 \%$.

Recurrence rate was significant to resection extent, according to Simpson grading system $(\mathrm{p}<0.01)$. For cases with total resection a recurrence rate of $13.8 \%$ was observed. Patients with incomplete resection had an overall recurrence rate of $46.7 \%$ (grade $2: 29.6 \%$, grade 3 : $56.3 \%$, grade $4: 51.3 \%$, grade $5: 100 \%$ ).

Our results show significant decline compared to Simpson's recurrence rates for each resection grade. We may only suppose that the operation notes of our institute's surgeons were not exact and that surgeons overestimated the extent of tumor removal, for each case. On the other hand, as Table 7 shows, in comparison with other studies, our series have similar recurrence rates.

Tumor site was not significant to meningioma's reappearance $(p>0.01)$, with the exception of certain locations whereas total resection was impossible. Tumors at these locations were very seldom.

Tumor histology was not a significant predictive factor for recurrence ( $p>0.01)$, with the exception of grade 2 and 3 meningiomas. For cases with atypical or malignant tumors separately, which accounted for $9.5 \%$ of all cases, histology was significant to recurrence. They presented with $41.6 \%$ and $75 \%$ percentage of recurrence, respectively. The morbidity and mortality outcomes were lower for grades 2 and 3 meningiomas.

Patient's sex and age were not related with recurrence. Females represented $62.3 \%$ of the cohort of our cases.

The rate of recurrence diminished with time from operation. Within 5 years from surgery, $94.7 \%$ of tumor reappearances were observed. This could suggest that in

Table 7. Comparing the recurrence rate between various studies.

\begin{tabular}{cc}
\hline Study & Total recurrence rate \\
\hline Ayerbe et al. & $21 \%$ \\
Jaaskelainen & $15.4 \%$ \\
Papanikolaou Hospital series & $21.5 \%$ \\
\hline
\end{tabular}


cases with total resection, recurrence is not often. In addition to that, in cases with non benign tumor, recurrence happens shortly after surgery.

Recurrence was an adverse prognostic factor for morbidity and mortality. Patients who recurred had reduced survival rates and $38.9 \%$ died because of tumor related factors.

\section{REFERENCES}

[1] D. Simpson, "The Recurrence of Intracranial Meningiomas after Surgical Treatment," Journal of Neurology Neurosurgery Psychiatry, Vol. 20, No. 22, 1957, pp. 11-21.

[2] D. Louis, H. Budka aand A. Von Deimling, "Meningiomas, Pathology and Genetics," Tumors of the Nervous System, IARC Press, Lyon, 1997, pp. 134-141.

[3] F. Yamasaki, H. Yoshioka, S. Hama, et al., "Recurrence of Meningiomas," Cancer, Vol. 89, No. 5, 1989, pp. $1102-1110$.

doi:10.1002/1097-0142(20000901)89:5<1102::AID-CNC $\underline{\text { R20>3.0.CO;2-L }}$

[4] H. Kamitani, H. Masuzawa, I. Kanazawa, et al., "Recurrence of Convexity Meningiomas: Tumor Cells in the Arachnoid Membrane," Surgery Neurology, Vol. 56, No. 4, 2001, pp. 228-235. doi:10.1016/S0090-3019(01)00582-1

[5] B. Borovich and Y. Doron, "Recurrence of Intracranial
Meningiomas: The Role Played by Regional Multicentricity," Journal of Neurosurgery, Vol. 64, No. 58, 1986, pp. 58-63. doi:10.3171/jns.1986.64.1.0058

[6] R. Mantle, B. Lach and M. Delgado, "Predicting the Probability of Meningioma Recurrence Based on the Quantity of Peritumoral Brain Edema on Computerized Tomography," Journal of Neurosurgery, Vol. 91, No. 3, 1999, pp. 375-383. doi:10.3171/jns. 1999.91.3.0375

[7] J. Ayerbe, R. Lobato, J. de la Cruz J, et al., "Risk Factors Predicting Recurrence in Patients Operated on for Intracranial Meningioma: A Multivariate Analysis, Acta Neurochirurgica, Vol. 141, No. 9, 1999, pp. 921-932. doi:10.1007/s007010050398

[8] K. Lee, T. Hoshiro and L. Rodriguez, "Bromodeoxyuridine Labeling Study of Intracranial Meningiomas: Proliferative Potential and Recurrence," Acta Neuropathology, Vol. 80, 1990, pp. 311-317. doi:10.1007/BF00294650

[9] H. Kolles, I. Niedermayer, C. Schmitt, et al., "Triple Approach for Diagnosis and Grading of Meningiomas: Histology, Morphometry of Ki-67/Feulgen Stainings and Cytometrics," Acta Neurochirurgica, Vol. 137, 1995, pp. 174-181. doi:10.1007/BF02187190

[10] J. Jaaskelainen, M. Haltia and A. Servo, "Atypical and Anaplastic Meningiomas: Radiology, Surgery, Radiotherapy and Outcome," Journal of Surgery Neurology, Vol. 25, 1986, pp. 233-242. doi:10.1016/0090-3019(86)90233-8 